\title{
SOME RESULTS ABOUT THE CONNECTIVITY OF TREES
}

\author{
Lilian Markenzon ${ }^{1}$, Nair Maria Maia de $\mathrm{Abreu}^{2 *}$ and Luciana Lee ${ }^{3}$
}

Received October 8, 2012 / Accepted March 15, 2013

\begin{abstract}
The second smallest Laplacian eigenvalue of a graph $G$ is called algebraic connectivity, denoted $a(G)$. The ordering of trees via this graph invariant is frequently studied in the literature. In this paper, we present a new invariant, the Internal Degree Sequence (IDS), that also supports an accurate evaluation of the connectivity of trees. We compare the $I D S$ with $a(G)$ for all elements in six classes of trees known to have the largest algebraic connectivity and we show that the IDS provides a strict total ordering of the elements of these classes. This result is also proved for a subclass of trees of diameter 4 .
\end{abstract}

Keywords: trees; internal degree sequence, algebraic connectivity.

\section{PRELIMINARIES}

Let $G=(V, E)$ be a simple graph, $n=|V|$ its order and $m=|E|$ its size. The degree of a vertex $v$ is denoted by $d(v)$. A graph is connected if for any two vertices $u, v \in V$ there is a path from $u$ to $v$. The graph is said to be disconnected if it has two or more connected components. The cut-vertex (or cutpoint) is a vertex whose removal increases the number of components of $G$.

The Laplacian matrix $L(G)=\left[\ell_{i j}\right]$ is defined as follows: $\ell_{i j}=d_{i}$, for $i=j ; \ell_{i j}=-1$ if $\left(v_{i}, v_{j}\right)$ is an edge of $G$ and $\ell_{i j}=0$ otherwise. The spectrum of the Laplacian of $G$ is the sequence of their eigenvalues $\left(\mu_{1}, \ldots, \mu_{n-1}, \mu_{n}\right)$, given in non-increasing order, $\mu_{1} \geq \cdots \geq \mu_{n-1} \geq \mu_{n}$. Since $L(G)$ is a semidefinite positive singular matrix, $\mu_{n}=0$. It is well known that $\mu_{n-1}=0$ if and only if $G$ is a disconnected graph. Due to that, Fiedler [3] called $\mu_{n-1}$ the algebraic connectivity of $G$, denoted $a(G)$. For a survey about $a(G)$ see Abreu [1]. The relation between the algebraic connectivity and the cutpoints of a graph has been addressed by Kirkland [6].

In this paper, we introduce a new concept for the connectivity of trees, the Internal Degree Sequence $(I D S)$, taking into account the number of connected components obtained after the

\footnotetext{
*Corresponding author

${ }^{1}$ NCE - Universidade Federal do Rio de Janeiro, Brazil. E-mail: markenzon@nce.ufrj.br

${ }^{2}$ Programa de Engenharia de Produção/COPPE, Universidade Federal do Rio de Janeiro. E-mail: nair@pep.ufrj.br

${ }^{3}$ Universidade Federal do Mato Grosso. E-mail: lucianalee@ufmt.br
} 
removal of each cut-vertex. An ordering for trees with $n$ vertices according to their $I D S$ is defined. In Section 3, we compare the $I D S$ with the algebraic connectivity $a(G)$ for all elements in six classes of trees known to have the largest algebraic connectivity, presented by Yuan et $a l$. [8], and we show that the $I D S$ provides a strict total ordering of the elements of these classes. This result is also proved for a subclass of trees of diamenter 4 in Section 4.

\section{THE NEW INVARIANT}

In this section, we present a new invariant, the internal degree sequence (IDS), taking into account the number of connected components obtained after the removal of each cut-vertex of the tree. The $I D S$ allows us to differentiate the connectivity of trees, providing an extra resource to compare them. Another important feature to highlight is the simplicity of the determination of the invariant.

The degree sequence of a graph is the non-increasing sequence of its vertex degrees. Let $T=$ $(V, E)$ be a tree and $\left[d_{1}, d_{2}, \ldots, d_{n}\right]$ its degree sequence. Let $V=\left\{v_{1}, v_{2}, \ldots, v_{n}\right\}$ be the ordered set of vertices of $T$, being $d_{i}=d\left(v_{i}\right)$.

A vertex $v$ of $T$ such that $d(v)>1$ is called an internal vertex of $T$. It is a cut-vertex of $T$. A vertex $v$ such that $d(v)=1$ is called a leaf or an external vertex of $T$.

Let $T=(V, E)$ be a tree and let $L \subset V$ be the set of leaves of $T$. The removal of a vertex $v \in V-L$ results in a disconnected tree; observe that there are $d(v)$ components in the resulting tree. It is not difficult to see that the degree of an internal vertex is directly related with its relevance in the analysis of the connectivity. The next definition takes this fact into consideration.

Definition 1. The non-increasing sequence of degrees of the internal vertices of a tree $T$ is called the internal degree sequence (IDS) of a tree T. It is denoted

$$
I D S(G)=\left[d_{1}, d_{2}, \ldots, d_{p}\right]
$$

Definition 2. Let $s=\left[d_{1}, d_{2}, \ldots, d_{p}\right]$ be a non-increasing sequence of integers; $s$ is a valid I $D S$ for a tree $T$ if $T$ has $n=\sum_{i=1, p} d_{i}-p+2$ vertices, $p$ internal vertices and $d_{i} \neq 1,1 \leq i \leq p$.

It is known that a string $t$ is preceded by a string $s$ in lexicographic order if

$-s$ is a prefix of $t$, or

- if $c$ and $d$ are respectively the first characters of $s$ and $t$ in which $s$ and $t$ differ, then $c$ precedes $d$ in character order.

It is possible to perform a lexicographic comparison of the $I D S$ values of two trees. For instance, Figure 1 shows all trees with 7 vertices with their respective $I D S$ in lexicographic order.

This example shows some coincidences with the algebraic connectivity that must be highlighted. Among all trees, the maximum and the minimum IDS occur with the star and the path, respectively; the same happens with the algebraic connectivity (Grone \& Merris [5]). This observation leads us to compare the two approaches, as we will see in the next section. 


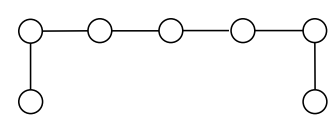

$[2,2,2,2,2]$

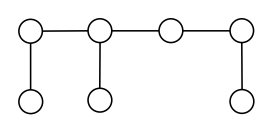

$[3,2,2,2]$

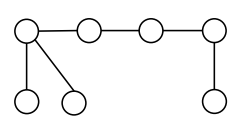

$[3,2,2,2]$

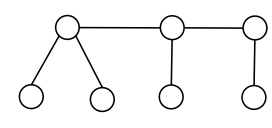

$[3,3,2]$

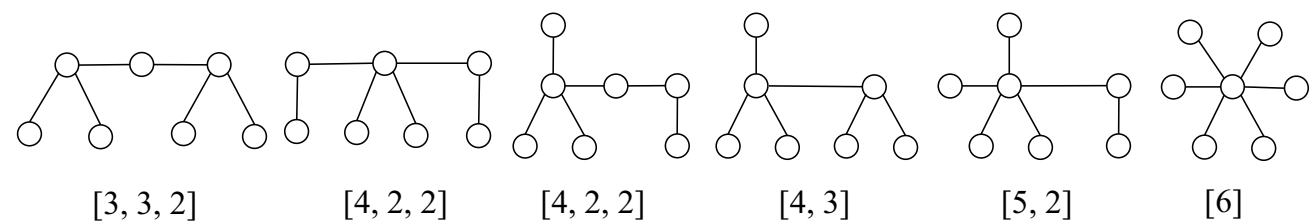

Figure 1 - All trees with $n=7$ and their respective IDS.

It is possible to generate all valid $I D S$ for a tree with $n$ vertices. The sequences can be build recursively, computing each element of the sequence from the first one. Proposition 1 provides some necessary results to build the sequences.

Proposition 1. Let $T$ be a tree with order $n$ and $I D S(T)=\left[d_{1}, \ldots, d_{p}\right]$ its internal degree sequence. Let $S=\sum_{i=1}^{p} d_{i}$. Then

1. the maximum value for $d_{i}$ is

$$
d_{i}= \begin{cases}n-p, & i=1 \\ \min \left\{\left(S-\sum_{j=1}^{i-1} d_{j}\right)-2(p-i), d_{i-1}\right\}, & 1<i \leq p\end{cases}
$$

2. the minimum value for $d_{i}$ is

$$
d_{i}= \begin{cases}\left\lceil\frac{S}{p}\right\rceil, & i=1 \\ \left\lceil\frac{S-\sum_{j=1}^{i-1} d_{j}}{p-(i-1)}\right\rceil, & 1<i \leq p .\end{cases}
$$

Proof. Let $T$ be a tree of order $n$ and let $\left[d_{1}, d_{2}, \ldots, d_{p}\right]$ be the $I D S$ of $T$. It is known that the sum of the degrees of the vertices of $V$ is $2(n-1)$. The sum of the degrees of the $p$ internal vertices of $T$ is $S=\sum_{i=1}^{p} d_{i}=n+p-2$.

1. The maximum degree of the first vertex of the sequence occurs when all the others have the minimum degree for an internal vertex (i.e. 2). Since $S=n+p-2$, the first element $d_{1}$ has value $S-2(p-1)=n-p$.

When computing $d_{i}, 1<i \leq p$, the previous elements $d_{1}, \ldots, d_{i-1}$ are already determined. It is possible to consider a sequence $\left[d_{i}, \ldots, d_{p}\right]$ with sum of degrees $S-$ $\sum_{j=1}^{i-1} d_{j}$. So, applying the previous reasoning and considering that, by definition, $d_{i} \leq$ $d_{i-1}$, the result is obtained. 
2. By Definition $1, d_{1} \geq \ldots \geq d_{p}$. In order to force $d_{1}$ to be minimum, the remaining degrees of $S$ must be scattered in other positions of the sequence, obtaining the value $S / p$. If the remainder is zero then $d_{1}=S / p$. Otherwise, let $r$ be the remainder. The positions $d_{1}, d_{2}$, $\ldots, d_{r}$ will have the value $S / P+1$. The minimum value of $d_{1}$ is $\lceil S / p\rceil$.

As in item(1), we consider $\left[d_{1}, \ldots, d_{i-1}\right]$ already determined, and the sum of the degrees $S-\sum_{j=1}^{i-1} d_{j}$. So, the result is obtained.

The next proposition shows how any sequence realizes a tree that obeys a specific condition.

Proposition 2. Given a non-increasing sequence of integers $s=\left[d_{1}, d_{2}, \ldots, d_{p}\right], d_{i} \neq 1$, there exists always a tree $T$ with $n=\sum_{i=1}^{p} d_{i}-p+2$ vertices such that $\operatorname{ID} S(T)=s$.

Proof. The proof is constructive. By the definition of a valid sequence it is known that $T$ has $n=\sum_{i=1}^{p} d_{i}-p+2$ vertices and $p$ internal vertices. The following steps build iteratively the tree $T$ :

- Step 1: Let $T$ be a tree with a central vertex $v$ and $d_{1}$ vertices,

$$
w_{1}, \ldots, w_{d_{1}} \text {, all adjacent to } v \text {. }
$$

- Step 2: For $i=2, \ldots, p$ do

Add $d_{i}-1$ new vertices, all adjacent to a leaf $w_{j}$ of $T$.

The graph $T$, resulting from Step 1, is a tree; as so, it has at least two leaves. Step 2 adds, at each iteration, $d_{i}-1$ new vertices, all adjacent to the same vertex $w_{j}$. At this point, vertex $w_{j}$ is no more a leaf, but at least one new leaf is created, maintaining the graph $T$ as a tree. So, tree $T$ has $d_{1}+\sum_{i=2, p}\left(d_{i}-1\right)$ leaves and $p$ internal vertices.

It is interesting to note that if the leaf $w_{j}$ chosen in Step 2 is the most recently added leaf, the resulting tree is a caterpillar (a tree such that if all leaves and their incident edges are removed, the remainder of the graph forms a path).

Since the lexicographic order provides a total ordering for all the valid sequences of graphs with a given $n$, the proof of the following proposition is immediate.

Proposition 3. The invariant I DS provides a total ordering for trees with $n$ vertices.

\section{I DS AND ALGEBRAIC CONNECTIVITY}

In 1987, Grone \& Merris [4] started an investigation about connectivity of trees through $a(G)$, proving in [5] that it is possible to present a strict total ordering of trees with diameter 3. After that, a large number of results comparing trees via $a(G)$ have appeared in the literature as, for instance, Fallat \& Kirkland [2] that extend the results of [5]. In this section, we compare some of the known results with those obtained using $I D S$. 
Proposition 4. [2] Let $P_{d-1}$ be a path with length $d-2$ labeled from 1 to $d-1$. Let $T_{d}(k, \ell)$ be a tree with $n$ vertices and diameter $d$, obtained from $P_{d-1}$ by adding $k$ pendant vertices adjacent to vertex 1 and $\ell$ pendant vertices adjacent to vertex $d-1$. Then

$$
a\left(T_{d}(k, \ell)\right)<a\left(T_{d}(k-1, \ell+1)\right), 1 \leq k \leq\left\lfloor\frac{n-d+1}{2}\right\rfloor .
$$

The internal vertices of $T_{d}(k, \ell)$ are the vertices of the path. So, the $I D S$ of $T_{d}(k, \ell)$ has $d-1$ elements and can be easily determined:

$$
\operatorname{IDS}\left(T_{d}(k, \ell)\right)=[\ell+1, k+1, \underbrace{2,2, \ldots, 2}_{d-3}] .
$$

For this class, it is easy to prove that the ordering provided by $I D S$ is a strict total ordering. Moreover, the orders given by $a(G)$ and $I D S$ are isomorphic. However, this behavior of the two measures does not occur in general.

Yuan et al. [8] introduced six classes of trees and show that, for $n \geq 15, a\left(T_{i}\right)>a\left(T_{j}\right)$ if $1 \leq i<j \leq 6$ and $T_{i}$ is any tree in the class $C_{i}$ and $T_{j}$ is any tree in the class $C_{j}$. These classes are defined below and we show the $I D S$ of their elements.

Definition 3. Let $n, k, p$ and $q$ be nonnegative integers with $3 k+2 p+q=n-1$. Let $T(k, p, q)$ be the tree of order $n$ which contains a vertex $v$ such that $T(k, p, q)-v=k K_{1,2} \cup p K_{1,1} \cup q K_{1}$.

Let $\mathcal{T}$ be the class of all trees $T(k, p, d)$. The following subclasses of $\mathcal{T}$ were defined in [8]:

$$
\begin{aligned}
& -C_{1}=\{T(0,0, n-1)\} ; \\
& -C_{2}=\{T(0,1, n-3)\} ; \\
& -C_{3}=\{T(0, p, q) \mid p \geq 2\} ; \\
& -C_{4}=\{T(1,0, n-4)\} ; \\
& -C_{5}=\{T(1, p, q) \mid p \geq 1\} ; \\
& -C_{6}=\{T(k, p, q) \mid k \geq 2\} .
\end{aligned}
$$

Notice that $\{T(k, p, q) \mid n=3 k+2 p+q+1\}=\cup_{i=1,6} C_{i}$.

For each $n$, classes $C_{1}, C_{2}$ and $C_{4}$ consist of only one tree. The trees belonging to $C_{3}$ and $C_{6}$ have the same algebraic connectivity, proved by Zhang [9] and Yuan et al. [8], respectively. Two important results about the ordering of all these trees are also presented by Yuan et al. [8] in Theorem 1.

Proposition 5. If $T(k, p, q)$ is any tree in class $C_{3}$, then $a(T)=(3-\sqrt{5}) / 2$.

Proposition 6. If $T(k, p, q), k \geq 2$, is any tree in class $C_{6}$, then $a(T)=2-\sqrt{3}$.

Theorem 1. Let $T_{i}$ be a tree in class $C_{i}$ for $i=1, \ldots, 6$. Then: 
1. For $n \geq 8, a\left(T_{1}\right)>a\left(T_{2}\right)>a\left(T_{3}\right)>a\left(T_{4}\right)>a\left(T_{5}\right)>a\left(T_{6}\right)$;

2. For $n \geq 15$ and $T \notin \cup_{i=1}^{6} C_{i}, a(T)<a\left(T_{6}\right)=2-\sqrt{3}$.

For the six classes it is possible to establish the IDS of their elements. And more, since several trees have the same algebraic connectivity, the IDS provides a good insight to distinguish accurately between them.

Let $T_{i}$ be a tree such that $T_{i} \in C_{i}, n \geq 8$. So, we have,

$$
\begin{aligned}
& -I D S\left(T_{1}\right)=[n-1] ; \\
& -I D S\left(T_{2}\right)=[n-2,2] ; \\
& -I D S\left(T_{3}\right)=[n-p-1, \underbrace{2,2, \ldots, 2}_{p}], p \geq 2 ; \\
& -I D S\left(T_{4}\right)=[n-3,3] ; \\
& -I D S\left(T_{5}\right)=[n-p-3,3, \underbrace{2,2, \ldots, 2}_{p}], p \geq 1 ; \\
& -I D S\left(T_{6}\right)=[n-2 k-p-\underbrace{1,3,3, \ldots, 3}_{k}, \underbrace{2,2, \ldots, 2}_{p}], k \geq 2 .
\end{aligned}
$$

The next proposition presents the comparison of the trees belonging to the six classes taking into account their $I D S$.

Proposition 7. Let $T_{i}$ be any tree of order $n, n \geq 8$, such that $T_{i} \in C_{i}$. Then

1. $\operatorname{IDS}\left(T_{1}\right)>\operatorname{IDS}\left(T_{2}\right)>\operatorname{IDS}\left(T_{4}\right)$;

2. $\operatorname{IDS}\left(T_{4}\right)>\operatorname{IDS}\left(T_{3}\right)$;

3. $\operatorname{IDS}\left(T_{4}\right)>\operatorname{IDS}\left(T_{5}\right)$;

4. $I D S\left(T_{4}\right)>I D S\left(T_{6}\right)$.

\section{Proof.}

1. Each class $C_{1}, C_{2}$ and $C_{4}$ has just one element. It is immediate to state that

$$
\operatorname{IDS}\left(T_{1}\right)>\operatorname{IDS}\left(T_{2}\right)>\operatorname{IDS}\left(T_{4}\right) .
$$

2. The tree $T \in C_{3}$ with the greatest $I D S$ has $p=2$ and $I D S(T)=[n-4,2,2]$. As $\operatorname{IDS}\left(T_{4}\right)=[n-3,3]$,

$$
\operatorname{IDS}\left(T_{4}\right)>\operatorname{IDS}\left(T_{3}\right)
$$


3. Similar reasoning can be made for classes $C_{5}$ and $C_{6}$.

The tree $T^{\prime} \in C_{5}$ with the greatest $I D S$ has $p=1$. The tree $T^{\prime \prime} \in C_{6}$ with the greatest $I D S$ has $k=2$ and $p=0$. So, $I D S\left(T^{\prime}\right)=[n-4,3,2]$ and $I D S\left(T^{\prime \prime}\right)=[n-5,3,3]$. Comparing with the $I D S$ of $T_{4}$

$$
I D S\left(T_{4}\right)>I D S\left(T_{5}\right) \text { and } I D S\left(T_{4}\right)>I D S\left(T_{6}\right) .
$$

It is impossible to compare the trees belonging to classes $C_{3}, C_{5}$ and $C_{6}$ as a whole. Figure 2 shows how the $I D S$ of the elements of these classes depend only on the types of their subtrees.

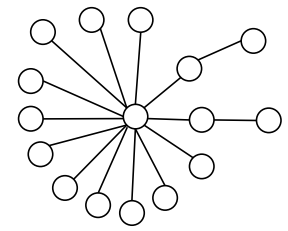

$T_{\mathrm{a}}(0,2,11) \in C_{3}$ $\operatorname{IDS}\left(T_{\mathrm{a}}\right)=[13,2,2]$

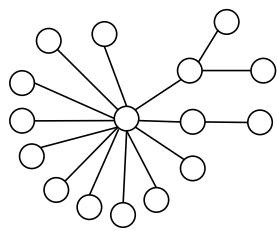

$T_{\mathrm{b}}(1,1,10) \in C_{5}$ $\operatorname{IDS}\left(T_{\mathrm{b}}\right)=[12,3,2]$

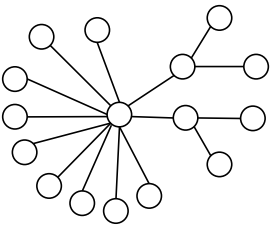

$T_{\mathrm{c}}(2,0,9) \in C_{6}$ $\operatorname{IDS}\left(T_{\mathrm{c}}\right)=[11,3,3]$

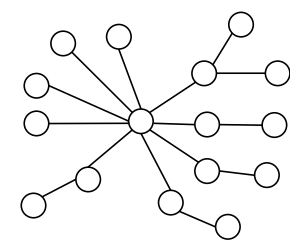

$T_{\mathrm{d}}(1,4,4) \in C_{5}$ $\operatorname{IDS}\left(T_{\mathrm{d}}\right)=[9,3,2,2,2,2]$

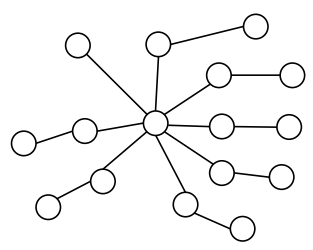

$T_{\mathrm{e}}(0,7,1) \in C_{3}$ $\operatorname{IDS}\left(T_{\mathrm{e}}\right)=[8,2,2,2,2,2,2,2]$

Figure 2 - Examples of trees belonging to classes $C_{3}, C_{5}$ and $C_{6}$.

However a very interesting result can be stated, showing an advantage of the invariant when the trees have the same algebraic connectivity.

Theorem 2. Given $n$, the I DS of the elements of the classes $C_{i}, i=1, \ldots, 6$, determine a strict total ordering of the trees $T(k, p, d)$.

Proof. We must prove that the trees belonging to $C_{3}, C_{5}$ and $C_{6}$ have different $I D S$ values, both within and among classes.

Let $T_{i}=T\left(k_{i}, p_{i}, q_{i}\right), T_{j}=T\left(k_{j}, p_{j}, q_{j}\right) \in C_{3}$. We know that, for $T_{3} \in C_{3}, \operatorname{IDS}\left(T_{3}\right)=$ $[n-p-1, \underbrace{2,2, \ldots, 2}_{p}], p \geq 2$. So,

$$
I D S\left(T_{i}\right)=I D S\left(T_{j}\right) \Longrightarrow p_{i}=p_{j}
$$


The order $n$ of $T_{i}$ and $T_{j}$ is given and $p_{i}=p_{j}$. As $n=2 p+q+1, q_{i}=q_{j}$ and the trees are the same and all trees in $C_{3}$ have different $I D S$ 's.

The proof is analogous when $T_{i}, T_{j} \in C_{5}$ and $T_{i}, T_{j} \in C_{6}$.

We must now show that trees belonging to different classes have different $I D S$. It is known that

$$
\begin{gathered}
I D S\left(T_{3}\right)=[n-p_{i}-1, \underbrace{2, \ldots, 2}_{p}], \\
I D S\left(T_{5}\right)=[n-p_{j}-3,3, \underbrace{2, \ldots, 2}_{p}] \text { and } \\
\operatorname{IDS}\left(T_{6}\right)=[n-2 k-p-1, \underbrace{3,3, \ldots, 3}_{k}, \underbrace{2,2, \ldots, 2}_{p}], k \geq 2 .
\end{gathered}
$$

Let $T \in C_{3}$. The tree $T$ cannot have the same $I D S$ as a tree belonging to $C_{5}$ or $C_{6}$ because the tree $T$ has no internal vertex of degree 3 which is mandatory in the two other classes.

Let $T^{\prime} \in C_{5}$. The tree $T^{\prime}$ cannot have the same $I D S$ as a tree belonging to $C_{6}$ because $T^{\prime}$ has exactly one internal vertex of degree 3 while the trees of $C_{6}$ have at least two vertices with such condition.

So, the invariant $I D S$ provides a strict total ordering for trees belonging to the classes $C_{i}, i=$ $1, \ldots, 6$.

\section{TREES WITH DIAMETER 4}

An important observation about the trees belonging to $\mathcal{T}$, presented in the previous section, is that all of them have diameter less or equal 4. The approach of looking at trees with small diameter was taken up again by Wang \& Tan [7]. They give continuity to the work of Yuan et al. [8], showing all trees of order $n \geq 45$ with algebraic connectivity in the interval $[(5-\sqrt{21}) / 2,2-\sqrt{3})$. The subclass of trees of diameter 4 is defined as follows by Zhang [10].

Definition 4. Let $p_{1}, p_{2}, \ldots, p_{k}$ be integers such that

$$
p_{1} \geq p_{2} \geq \ldots \geq p_{k} \geq 0, \quad p_{1} \geq p_{2}>0, k \geq 2, k+1+p_{1}+\ldots+p_{k}=n .
$$

Let $T\left(n, k, p_{1}, p_{2}, \ldots, p_{k}\right)$ be a tree with diameter $4, N(c)=\left\{v_{1}, \ldots, v_{k}\right\}$, being $c$ the unique center of the tree, $d\left(v_{1}\right)=p_{1}+1, \ldots, d\left(v_{k}\right)=p_{k}+1$. Let $\mathbf{T}$ be the class of all trees $T\left(n, k, p_{1}, p_{2}, \ldots, p_{k}\right)$.

Wang \& Tan[7] defined new subclasses of trees as follows:

- $C_{1}^{\prime}=\{S(3, n-5)\}$, the tree of order $n$ obtained by joining the center of $K_{1,3}$ to the center of $K_{1, n-5}$ with an edge;

$$
-C_{2}^{\prime}=\left\{T\left(n, k, 3,1, p_{3}, \ldots, p_{k}\right)\right\}
$$




$$
\begin{aligned}
& -C_{3}^{\prime}=\left\{T\left(n, k, 3,2, p_{3}, \ldots, p_{k}\right)\right\} \\
& -C_{4}^{\prime}=\left\{T\left(n, k, 3,3, p_{3}, \ldots, p_{k}\right)\right\}
\end{aligned}
$$

Notice that $C_{1}^{\prime}$ is not a subclass of $\mathbf{T}$.

Wang \& Tan [7] proved interesting results about these classes:

Proposition 8. Let $T$ be a tree of order $n \geq 45$. If $T \notin \mathcal{T} \cup\left(\cup_{i=1}^{4} C_{i}^{\prime}\right)$, then $a(T)<(5-\sqrt{21}) / 2$.

Theorem 3. If $T$ is a tree of order $n \geq 45$, then $(5-\sqrt{21}) / 2 \leq a(T)<2-\sqrt{3}$ if and only if $T \in \cup_{i=1}^{4} C_{i}^{\prime}$.

The authors were able to provide the three trees with the first, second and third largest algebraic connectivity in $T_{n} \backslash \mathcal{T}$. However few results are presented about the ordering of all trees with diameter 4 .

Using the IDS, an interesting result can be shown. We define a subclass of $\mathbf{T}$. Let $\mathbf{T}^{\prime}$ be the class of all $T\left(n, k, p_{1}, p_{2}, \ldots, p_{k}\right)$ such that $k>p_{1}$, i.e., the set of all trees of diameter 4 such that the center of the tree has the greatest degree. It is possible to determine the $I D S$ of a tree $T \in \mathbf{T}^{\prime}$ directly from its notation.

Definition 5. Let $T\left(n, k, p_{1}, \ldots, p_{z}, \ldots, p_{k}\right)$ with $k>p_{1}$. Let $p_{z}, 2 \leq z \leq k$, be the last non-zero element of the notation. Then

$$
\operatorname{IDS}(T)=\left[k, p_{1}+1, p_{2}+1, \ldots, p_{z}+1\right] .
$$

Theorem 4. Given $n$, the IDS of the elements of the class $\mathbf{T}^{\prime}$ determine a strict total ordering of the trees $T\left(n, k, p_{1}, p_{2}, \ldots, p_{k}\right)$ with $k>p_{1}$.

Proof. Let $T\left(n, k, p_{1}, p_{2}, \ldots, p_{k}\right) \in \mathbf{T}^{\prime}$. Let $S$ be the sequence $\left[n, k, p_{1}, p_{2}, \ldots, p_{z}, \ldots, p_{k}\right]$. It is immediate to conclude that $S$ is a non-increasing sequence. The sequence $\operatorname{IDS}(T)$ is build from $S$ and its elements obey exactly the original ordering. So, given $n$, there is a unique tree with this sequence as $I D S$.

Analogous result can be shown for all trees of diameter 4 such that the central vertex has the smallest degree.

\section{ACKNOWLEDGMENTS}

This work was supported by grants 305372/2009-2, 305016/2006-7, CNPq, Brazil and project 027/CAP/2011, Universidade Federal do Mato Grosso, Brazil.

\section{REFERENCES}

[1] ABREU N. 2007. Old and new results on algebraic connectivity of graphs. Linear Algebra and its Applications, 423: 53-73. 
[2] FALLAT S \& KiRKLAND S. 1998. Extremizing algebraic connectivity subject to graph theoretical constrains. Electronic Journal Linear Algebra, 3: 48-74.

[3] Fiedler M. 1973. Algebraic connectivity of graphs. Czechoslovak Mathematical Journal, 23: 298-305.

[4] Grone R \& Merris R. 1987. Algebraic connectivity of trees. Czechoslovak Mathematical Journal, 37: $660-670$.

[5] Grone R \& MERRIS R. 1990. Ordering trees by algebraic connectivity. Graphs and Combinatorics, 6: $229-237$.

[6] KiRKLAND S. 2001. Un upper bound on algebraic connectivity of graphs with many cutpoints. Electronic Journal Linear Algebra, 8: 94-109.

[7] WANG X-K \& TAN S-W. 2012. Ordering trees by algebraic connectivity. Linear Algebra and its Applications, 436: 3684-3691.

[8] YUAN X, SHaO J \& ZHANG L. 2008. The six classes of trees with the largest algebraic connectivity. Discrete Applied Mathematics, 156: 757-769.

[9] Zhang XD. 2004. On the two conjectures of Graffiti. Linear Algebra and its Applications, 385: 369-379.

[10] ZHANG XD. 2007. Ordering trees with algebraic connectivity and diameter. Linear Algebra and its Applications, 427: 301-312. 